\title{
Design and Evaluation of Three-dimensional Zig-zag Chaotic Micromixers for Biochemical Applications
}

Xingjian Hu ${ }^{a}$, Fan Yang ${ }^{a}$, Haiyan Zhao ${ }^{a,{ }^{*}}$, Mingzhao Guo ${ }^{b}$, Yujun Wang ${ }^{b,{ }^{*}}$

a. State Key Laboratory of Tribology, Department of Mechanical Engineering, Tsinghua University, Beijing 100084, China

b. State Key Laboratory of Chemical Engineering, Department of Chemical Engineering, Tsinghua University, Beijing 100084, China

* Corresponding author. E-mail address: hyzhao@tsinghua.edu.cn (H. Zhao) and wangyujun@tsinghua.edu.cn (Y. Wang).

\section{Supporting Information}

\section{Section S1 Solver introduction, mesh dependency test and model validation}

In this simulation, the solver is stationary and couples laminar flow (spf) and transport of diluted species (tds) physics interfaces. The convergence criteria is 0.001 relative tolerance. The free tetrahedral mesh was exploited for the numerical model. A mesh dependency test was carried out to seek the optimal grid size, minimizing the computing time and guaranteeing the solution accuracy simultaneously. Five mesh models with grid numbers ranging from 61100 to 1405582 were applied and tested in $180 \mathrm{~T}+60 \mathrm{~W}$ micromixer at $R e=30$. The mixing index of the cross-section along the mixing distance 
was studied, and its error was calculated based on the solution of the 1405582-element model. The result is shown in Figure S1. Obviously, the solutions of the 1015665element model are very close to the 1405582-element, and the maximum error is below $10 \%$, especially at $0.65 \mathrm{~mm}$ mixing distance. Therefore, 1015665 was selected as the optimal mesh number with element size ranging from $\sim 0.002 \mathrm{~mm}$ to $\sim 0.02 \mathrm{~mm}$. Other numerical models in this paper have also undergone the same mesh dependency test.

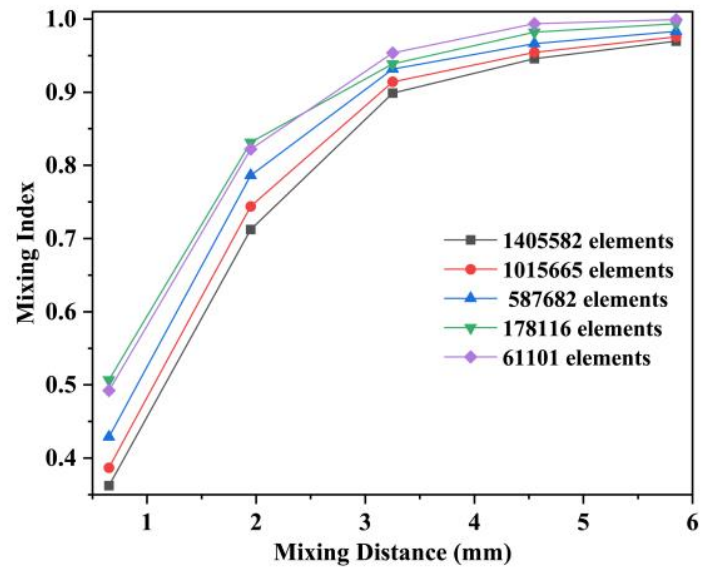

(a)

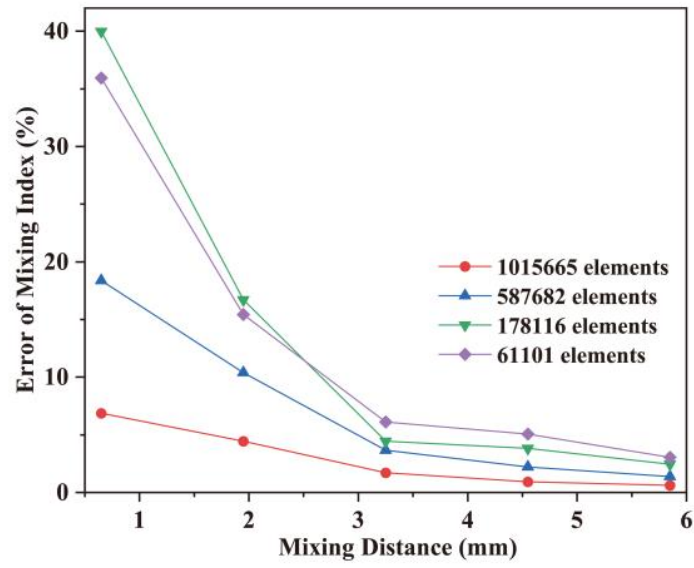

(b)

Figure S1. Mesh dependency test results: (a) mixing indices at different mixing distance with mesh numbers ranging from 61101 to 1405582; (b) error of mixing indices compared with the 1405582-element model.

In order to validate the reliability of numerical simulation, we compared the previous experimental results (Chen et al. ${ }^{1}$ ) with the simulation results obtained from the numerical model with similar parameter settings in this paper. As is shown in Figure S2, the concentration fraction distribution of sodium hydroxide and phenolphthalein in numerical contours agrees well with experimental micrographs, proving the accuracy of numerical calculation. 

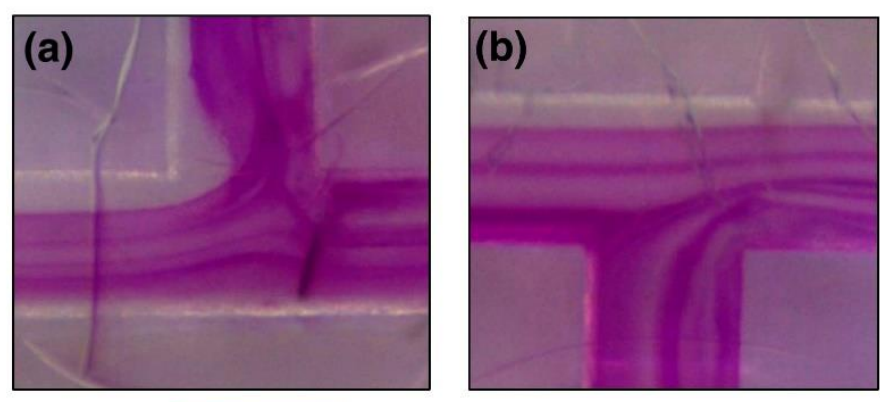

Mass

Fraction
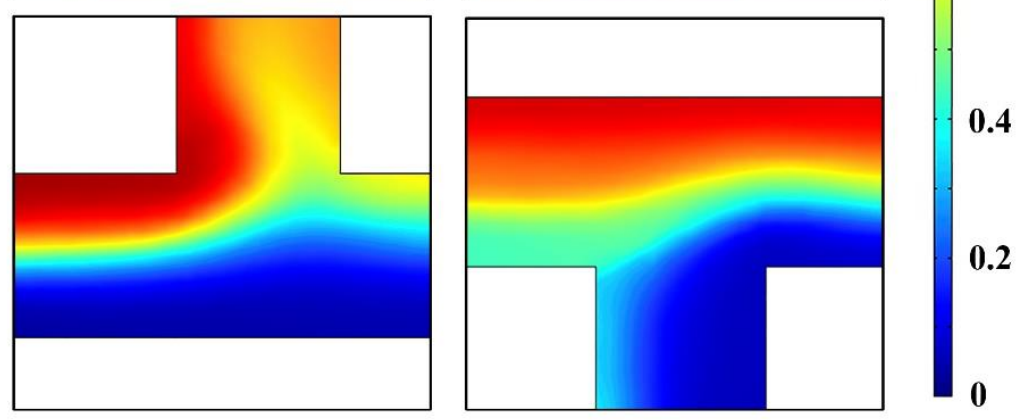

Figure S2. Concentration fraction distribution comparison between previous experimental results (upper row, Reprinted by permission from Ref. 1, Springer Nature Copyright 2016) and present numerical simulation results (lower row, conducted with similar model parameter settings in this work).

\section{Section S2 Villermaux-Dushman method and segregation index}

Villermaux-Dushman (V-D) method $^{2}$ is based on the iodide-iodate reaction system, which is shown below:

$$
\begin{gathered}
\mathrm{H}_{2} \mathrm{BO}_{3}^{-}+\mathrm{H}^{+} \rightarrow \mathrm{H}_{3} \mathrm{BO}_{3} \text { (quasi-instaneous) } \\
5 \mathrm{I}^{-}+\mathrm{IO}_{3}^{-}+6 \mathrm{H}^{+} \rightarrow 3 \mathrm{I}_{2}+3 \mathrm{H}_{2} \mathrm{O} \text { (fast, but much slower than Reaction S1) } \\
\mathrm{I}^{-}+\mathrm{I}_{2} \leftrightarrow \mathrm{I}_{3}^{-}
\end{gathered}
$$

where kinetic parameters for Reaction S3 at 298.15 K can be specified as:

$$
r_{3}=k_{3}\left[\mathrm{I}_{2}\right]\left[\mathrm{I}^{-}\right]-k_{3}^{\prime}\left[\mathrm{I}_{3}^{-}\right], k_{3}=5.9 \times 10^{9} \mathrm{~m}^{3} /(\mathrm{kmol} \cdot \mathrm{s}), k_{3}^{\prime}=7.5 \times 10^{9} / \mathrm{s}
$$

Also, the equilibrium constant $K_{3}$ for Reaction $\mathbf{S 3}$ is defined below: 


$$
K_{3}=k_{3} / k_{3}^{\prime}=\left[\mathrm{I}_{3}^{-}\right] /\left[\mathrm{I}_{2}\right]\left[\mathrm{I}^{-}\right]
$$

wherein $K_{3}$ solely depends on temperature so that it can also be expressed as:

$$
\lg K_{3}=555 / T+7.355-2.575 \lg T
$$

In this method, Reaction S1 and $\mathbf{S 2}$ are competing for $\mathrm{H}^{+}$in the system, and the product will solely depend on the kinetic parameters of reactions if micromixing of reactant solutions is perfect. According to the reaction rates of Reaction S1 and S2, inflow $\mathrm{H}^{+}$will be consumed by $\mathrm{H}_{2} \mathrm{BO}_{3}^{-}$instantaneously and completely so that there will be no $\mathrm{I}_{2}$ yielded in the system when micromixing thoroughly. On the contrary, if $\mathrm{H}^{+}$is locally over-concentrated due to slow micromixing, $\mathrm{H}^{+}$will have more chances to react with $\mathrm{I}^{-}$and $\mathrm{IO}_{3}^{-}$, and yield $\mathrm{I}_{2}$ after complete neutralization of local $\mathrm{H}_{2} \mathrm{BO}_{3}^{-}$. Furthermore, $\mathrm{I}_{2}$ will undergo Reaction $\mathbf{S 3}$ with $\mathrm{I}^{-}$and yield $\mathrm{I}_{3}^{-}$. The main composition of reactant solutions in the V-D experiment is shown in Table S1.

The segregation index $X_{S}$ is introduced to quantify the micromixing efficiency of micromixers based on the V-D method, which is defined as follows:

$$
X_{S}=Y / Y_{S T}
$$

where $Y$ is the mole ratio of the acid consumed by Reaction $\mathbf{S 2}$ to the total inflow acid. In this work, there are two inflows of reaction solutions so that the equation for $Y$ is:

$$
Y=\frac{2\left(Q_{A}+Q_{B}\right)\left(\left[\mathrm{I}_{2}\right]+\left[\mathrm{I}_{3}^{-}\right]\right)}{Q_{A}\left[\mathrm{H}^{+}\right]_{0}}
$$

where $Q_{A}$ and $Q_{B}$ are the average volumetric flow rates of Solution $\mathrm{A}$ and $\mathrm{B}$, respectively. $Y_{S T}$ is the value of $\mathrm{Y}$ concerning stoichiometric allocation when the micromixing process is infinitely slow. The equation for $Y_{S T}$ is shown below: 


$$
Y_{S T}=\frac{6\left[\mathrm{IO}_{3}^{-}\right]_{0}}{6\left[\mathrm{IO}_{3}^{-}\right]_{0}+\left[\mathrm{H}_{2} \mathrm{BO}_{3}^{-}\right]_{0}}
$$

The value of $X_{S}$ ranges from 0 to $1 . X_{S}=0$ represents the perfect micromixing state while $X_{S}=1$ represents the complete segregation state.

Table S1. The main composition of reactant solutions in the V-D experiment (reprinted with permission from ref. 3, Copyright 2019 ACS Publications)

\begin{tabular}{|c|c|c|c|c|}
\hline$(\mathrm{mol} / \mathrm{L})$ & $\mathbf{H}_{\mathbf{2}} \mathbf{B O}_{\mathbf{3}}^{-}$ & $\mathbf{I}^{-}$ & $\mathbf{I O}_{\mathbf{3}}^{-}$ & $\mathbf{H}^{+}$ \\
\hline Solution A & 0.0909 & 0.0118 & 0.00236 & $\backslash$ \\
\hline Solution B & $\backslash$ & $\backslash$ & $\backslash$ & $0.06^{*}$ \\
\hline
\end{tabular}

*Note: the concentration of $\mathrm{H}+$ refers to the value when acid is completely dissociated.

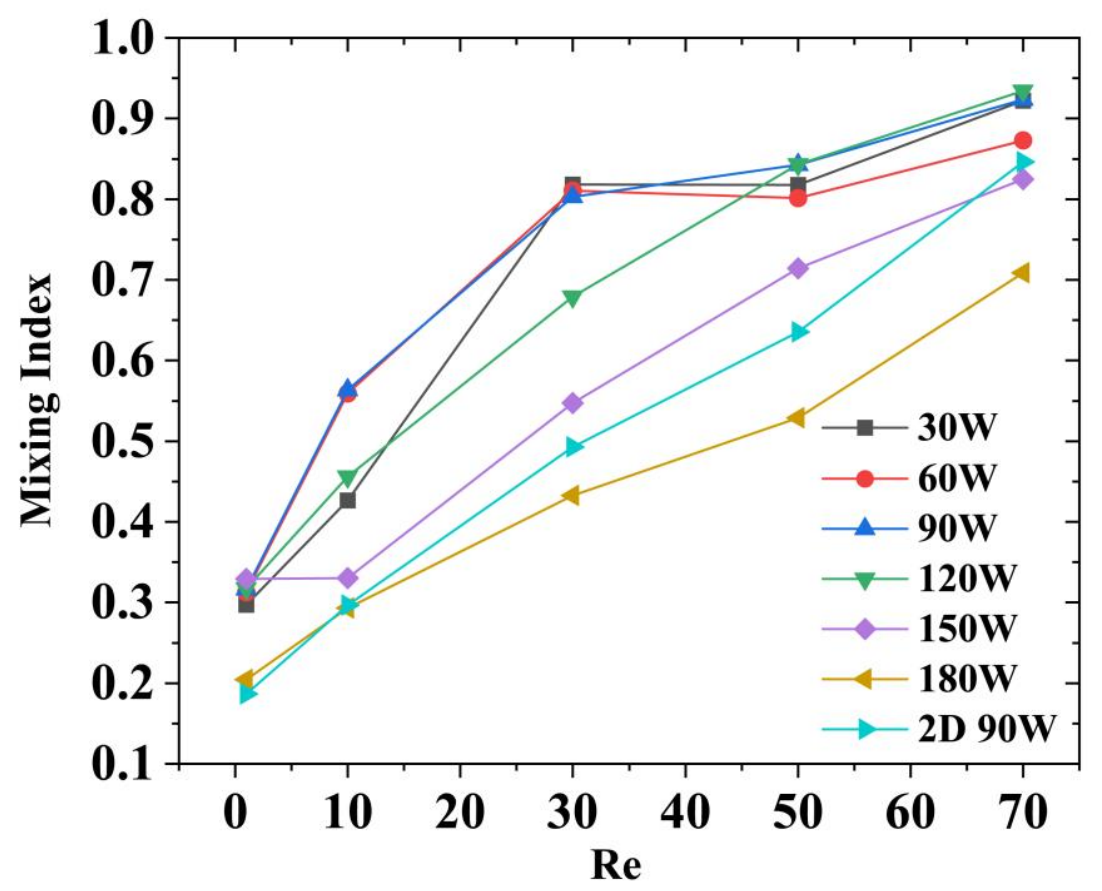

Figure S3. Mixing indices at outlets of micromixers summarized from Figure 5. 


\section{References}

1. Chen, X.; Shen, J., Numerical and experimental investigation on splitting-andrecombination micromixer with E-shape mixing units. Microsystem Technologies 2016, 23 (10), 4671-4677.

2. Fournier, M.-C.; Falk, L.; Villermaux, J., A new parallel competing reaction system for assessing micromixing efficiency—experimental approach. Chem. Eng. Sci. 1996, $51(22), 5053-5064$.

3. Guo, M.; Hu, X.; Yang, F.; Jiao, S.; Wang, Y.; Zhao, H.; Luo, G.; Yu, H., Mixing Performance and Application of a Three-Dimensional Serpentine Microchannel Reactor with a Periodic Vortex-Inducing Structure. Ind. Eng. Chem. Res. 2019, $58(29), 13357-13365$. 